\title{
Exotic phase separation and phase diagrams of a Fermi-Fermi mixture in a trap at finite temperature
}

\author{
Jibiao Wang, ${ }^{1}$ Hao Guo, ${ }^{2,3}$ and Qijin Chen ${ }^{1, *}$ \\ ${ }^{1}$ Department of Physics and Zhejiang Institute of Modern Physics, Zhejiang University, Hangzhou, Zhejiang 310027, China \\ ${ }^{2}$ Department of Physics, Southeast University, Nanjing 211189, China \\ ${ }^{3}$ Department of Physics, University of Hong Kong, Hong Kong, China
}

(Received 23 September 2012; revised manuscript received 12 January 2013; published 11 April 2013)

\begin{abstract}
The pairing and superfluid phenomena in a two-component Fermi gas can be strongly affected by the population and mass imbalances. Here we present phase diagrams of an atomic Fermi-Fermi mixture as they undergo BCS-Bose-Einstein condensation (BEC) crossover using a pairing fluctuation theory. We focus on the finite temperature and trap effects, with an emphasis on the mixture of ${ }^{6} \mathrm{Li}$ and ${ }^{40} \mathrm{~K}$. We show that there exist exotic types of phase separation in the (near-)BEC regime, associated with a pseudogap effect. Moreover, in the BCS and unitary regimes, the spatial density and gap profiles exhibit sandwichlike shell structures with superfluid or pseudogapped normal state in the middle shell. Such a sandwichlike shell structure appears when the mass imbalance increases beyond a certain threshold. Our result is relevant to future experiments on the ${ }^{6} \mathrm{Li}^{-}{ }^{40} \mathrm{~K}$ and other possible Fermi-Fermi mixtures.
\end{abstract}

DOI: 10.1103/PhysRevA.87.041601

PACS number(s): 03.75.Ss, 03.75.Hh, 67.85.Pq, 74.25.Dw

Ultracold Fermi gases provide an excellent model system for studying condensed matter physics, e.g., the pseudogap phenomena in high- $T_{c}$ superconductivity [1], owing to various experimentally tunable parameters. Using a Feshbach resonance, a population balanced two-component Fermi gas of equal mass exhibits a perfect crossover from a BCS type of superfluidity to Bose-Einstein condensation (BEC) $[2,3]$. There have been a great deal of experimental and theoretical studies on equal-mass systems with and without population imbalance [4,5]. In particular, population imbalance adds a new dimension to the phase diagrams, leading to phase separation [6], Sarma superfluid [7], and possibly FuldeFerrell-Larkin-Ovchinnikov (FFLO) states [8]. Mass imbalance, i.e., pairing of different mass atoms, will further enrich the physics, e.g., leading to significant trimer correlations under certain conditions [9], and thus make the subject very complex. Indeed, there have been worldwide efforts on the study of Fermi-Fermi mixtures of different species. Over the past several years, Feshbach resonances between different species of fermionic atoms, e.g., ${ }^{6} \mathrm{Li}$ and ${ }^{40} \mathrm{~K}$, have been found and studied [10-12], although it remains to achieve superfluidity experimentally. There have been some theoretical studies in this aspect, e.g., on the strong attraction limit at zero temperature $T$ [13], few-body physics [14], the polaron physics [15], as well as thermodynamics of a high $T$ normal mixture [16]. There have also been studies on phase diagrams, which, however, are mostly restricted to zero temperature using a mean-field theory either in a homogeneous Fermi gas $[17,18]$ or in a trap [19-21]. Recently, Guo et al. [22] as well as Stoof and co-workers [23] studied the mass imbalanced Fermi gases at finite temperatures. Due to technical complexity, this study was restricted to homogeneous cases. In order to address various experiments, which are always done at finite $T$ and in a trap, it is important to take into account the trap and finite $T$ effects simultaneously.

\footnotetext{
*Corresponding author: qchen@zju.edu.cn
}

In this Rapid Communication, we consider a two-species Fermi-Fermi mixture with a short-range $s$-wave pairing interaction in a three-dimensional (3D) isotropic harmonic trap at finite temperature. We emphasize on the interplay between the finite $T[4,24]$ and trap effects while the mass ratio $m_{\uparrow} / m_{\downarrow}$ and population imbalance (or "spin polarization") $p=$ $\left(N_{\uparrow}-N_{\downarrow}\right) /\left(N_{\uparrow}+N_{\downarrow}\right)$ (as well as the ratio $\omega_{\uparrow} / \omega_{\downarrow}$ between the trapping frequencies) are varied within a pairing fluctuation theory [25], where spin index $\sigma=\uparrow, \downarrow$ refers to the heavy and light species, respectively. In order to address experiments, we pay special attention to the ${ }^{6} \mathrm{Li}^{40} \mathrm{~K}$ mixture, while our result as a function of mass ratio covers other possible mixtures such as ${ }^{6} \mathrm{Li}^{1}{ }^{173} \mathrm{Yb}$ and ${ }^{171} \mathrm{Yb}^{173} \mathrm{Yb}$. One special feature of our theory is the emergence of widespread pseudogap phenomena [26-28] at finite $T$. The analogous pseudogap phenomena are of central importance in the field of high- $T_{c}$ superconductivity [1]. For high enough mass imbalance, we find that, in the $T-p$ phase diagrams, three-shell sandwichlike spatial structures occupy a large region both at unitarity and in the BCS regime, including sandwiched phase separated superfluid, sandwiched Sarma superfluid, and sandwiched polarized pseudogap states with increasing $T$. In the BEC regime, there are exotic "inverted" phase separations with a normal Fermi gas core in the trap center surrounded by paired (superfluid or pseudogap) states in the outer shell. Our result provides important predictions for future experiments.

Except for slightly different notations, our formalism is a combination of that used in Refs. [22,29], where a single-channel Hamiltonian is used to describe the Fermi gas, with a local density approximation (LDA) for addressing the trap inhomogeneity. The bare fermion Green's function is given by $G_{0 \sigma}^{-1}(K)=i \omega_{n}-\xi_{\mathbf{k} \sigma}$, with dispersion $\xi_{\mathbf{k} \sigma} \equiv \epsilon_{\mathbf{k} \sigma}-\mu_{\sigma}=k^{2} / 2 m_{\sigma}-\mu_{\sigma}$, chemical potential $\mu_{\sigma}$, and fermionic Matsubara frequency $\omega_{n}$. Here again, we take $\hbar=k_{B}=1$ and use the four-vector notation, e.g., $K \equiv$ $\left(\omega_{n}, \mathbf{k}\right), \sum_{K} \equiv T \sum_{n} \sum_{\mathbf{k}}$, etc. The self-energy $\Sigma_{\sigma}(K)=$ $\Sigma_{\mathrm{sc}, \sigma}(K)+\Sigma_{\mathrm{pg}, \sigma}(K)$ contains two parts, where the condensate contribution $\Sigma_{\mathrm{sc}, \sigma}(K)=-\Delta_{\mathrm{sc}}^{2} G_{0 \bar{\sigma}}(-K)$ vanishes above $T_{c}$ (with $\bar{\sigma}=-\sigma$ ), and the finite momentum pair contribution 
$\Sigma_{\mathrm{pg}, \sigma}(K)=\sum_{Q} t_{\mathrm{pg}}(Q) G_{0 \bar{\sigma}}(Q-K)$ persists down to $T=$ 0 . The $T$-matrix $t_{\mathrm{pg}}(Q)=g /[1+g \chi(Q)]$ represents an infinite series of particle-particle scattering processes, with a short-range interaction strength $g<0$ and the pair susceptibility $\chi(Q)=\sum_{K, \sigma} G_{0 \sigma}(Q-K) G_{\bar{\sigma}}(K) / 2$. For $T \leqslant T_{c}$, we have $\Sigma_{\mathrm{pg}, \sigma}(K)=\sum_{Q} t_{\mathrm{pg}}(Q) G_{0 \bar{\sigma}}(-K)+\delta \Sigma_{\sigma}=$ $-\Delta_{\mathrm{pg}}^{2} G_{0 \bar{\sigma}}(-K)+\delta \Sigma_{\sigma}$, which defines a pseudogap $\Delta_{\mathrm{pg}}$ via $\Delta_{\mathrm{pg}}^{2} \equiv-\sum_{Q} t_{\mathrm{pg}}(Q)$. Ignoring the less important incoherent term $\delta \Sigma_{\sigma}$, we obtain $\Sigma_{\sigma}(K)=-\Delta^{2} G_{0 \bar{\sigma}}(-K)$ in the simple BCS form, where $\Delta^{2}=\Delta_{\mathrm{sc}}^{2}+\Delta_{\mathrm{pg}}^{2}$. Therefore, the full Green's function is given by

$$
G_{\sigma}(K)=\frac{u_{\mathbf{k}}^{2}}{i \omega_{n}-E_{\mathbf{k} \sigma}}+\frac{v_{\mathbf{k}}^{2}}{i \omega_{n}+E_{\mathbf{k} \bar{\sigma}}},
$$

where $\quad u_{\mathbf{k}}^{2}=\left(1+\xi_{\mathbf{k}} / E_{\mathbf{k}}\right) / 2, \quad v_{\mathbf{k}}^{2}=\left(1-\xi_{\mathbf{k}} / E_{\mathbf{k}}\right) / 2, \quad E_{\mathbf{k}}=$ $\sqrt{\xi_{\mathbf{k}}^{2}+\Delta^{2}}$, and $E_{\mathbf{k} \sigma}=E_{\mathbf{k}}+\zeta_{\mathbf{k} \sigma}, \xi_{\mathbf{k}}=\left(\xi_{\mathbf{k} \uparrow}+\xi_{\mathbf{k} \downarrow}\right) / 2, \zeta_{\mathbf{k} \sigma}=$ $\left(\xi_{\mathbf{k} \sigma}-\xi_{\mathbf{k} \bar{\sigma}}\right) / 2$. With $n_{\sigma}=\sum_{K} G_{\sigma}(K), n=n_{\uparrow}+n_{\downarrow}$ and $\delta n=$ $n_{\uparrow}-n_{\downarrow}$, the number equations read

$$
\begin{gathered}
n=\sum_{\mathbf{k}}\left[\left(1-\frac{\xi_{\mathbf{k}}}{E_{\mathbf{k}}}\right)+2 \bar{f}\left(E_{\mathbf{k}}\right) \frac{\xi_{\mathbf{k}}}{E_{\mathbf{k}}}\right], \\
\delta n=\sum_{\mathbf{k}}\left[f\left(E_{\mathbf{k} \uparrow}\right)-f\left(E_{\mathbf{k} \downarrow}\right)\right],
\end{gathered}
$$

where the average Fermi function $\bar{f}(x) \equiv \sum_{\sigma} f\left(x+\zeta_{\mathbf{k} \sigma}\right) / 2$.

At $T \leqslant T_{c}$, the Thouless criterion leads to the gap equation $g^{-1}+\chi(0)=0$. For $T>T_{c}$, it is amended by $g^{-1}+\chi(0)=$ $Z \mu_{p}$, where the effective pair chemical potential $\mu_{p}$ and the coefficient $Z$ can be determined from the Taylor expansion of the inverse $T$ matrix [4], $t_{\mathrm{pg}}^{-1}(Q)=Z\left(i \Omega_{l}-\tilde{\Omega}_{\mathbf{q}}\right)$, with $\tilde{\Omega}_{\mathbf{q}}=$ $q^{2} / 2 M^{*}-\mu_{p}$ being the pair dispersion, and $M^{*}$ the effective pair mass. Thus the gap equation reads

$$
\frac{m_{r}}{2 \pi a}=\sum_{\mathbf{k}}\left[\frac{1}{2 \epsilon_{\mathbf{k}}}-\frac{1-2 \bar{f}\left(E_{\mathbf{k}}\right)}{2 E_{\mathbf{k}}}\right]+Z \mu_{p},
$$

with $\mu_{p}=0$ at $T \leqslant T_{c}$. Here $g$ is replaced via $g^{-1}=$ $m_{r} / 2 \pi a-\sum_{\mathbf{k}} 1 / 2 \epsilon_{\mathbf{k}}$, where $a$ is the $s$-wave scattering length, $m_{r}=m_{\uparrow} m_{\downarrow} /\left(m_{\uparrow}+m_{\downarrow}\right)$ the reduced mass, and $\epsilon_{\mathbf{k}}=$ $k^{2} / 4 m_{r}=\xi_{\mathbf{k}}+\mu$, with $\mu=\left(\mu_{\uparrow}+\mu_{\downarrow}\right) / 2$.

The $T$-matrix expansion leads to the pseudogap equation

$$
\Delta_{\mathrm{pg}}^{2}=Z^{-1} \sum_{\mathbf{q}} b\left(\tilde{\Omega}_{\mathbf{q}}\right),
$$

where $b(x)$ is the Bose distribution function. As in Ref. [22], we impose a cutoff $q_{c}$ on the summation such that pairs with $q>q_{c}$ may decay into the particle-particle continuum.

In the trap of frequency $\omega_{\sigma}$, the LDA approximation imposes that the local $\mu_{\sigma}(r)=\mu_{\sigma}(0)-\frac{1}{2} m_{\sigma} \omega_{\sigma}^{2} r^{2}$. We have the total particle number $N=\int d^{3} r n(r)$ and the number difference $\delta N=N_{\uparrow}-N_{\downarrow}=p N=\int d^{3} r \delta n(r)$. The Fermi energy $E_{F}=(3 N)^{1 / 3} \omega_{\uparrow}=k_{F}^{2} / 2 m=m R_{\mathrm{TF}}^{2} \omega_{\uparrow}^{2} / 2=T_{F}$ is defined as that for an unpolarized, noninteracting Fermi gas with the same total number $N$ and trap frequency $\omega_{\uparrow}$, with an equal mass $m=\left(m_{\uparrow}+m_{\downarrow}\right) / 2$. Here $R_{\mathrm{TF}}$ is the Thomas-Fermi radius, and the species dependent $R_{\mathrm{TF}}^{\sigma}=\sqrt{2\left(6 N_{\sigma}\right)^{1 / 3} / m_{\sigma} \omega_{\sigma}}$.

To find the stable states in a trap, we compare the (local) thermodynamical potential $\Omega_{S}$ (per unit volume) in a superfluid or pseudogap state with its normal Fermi

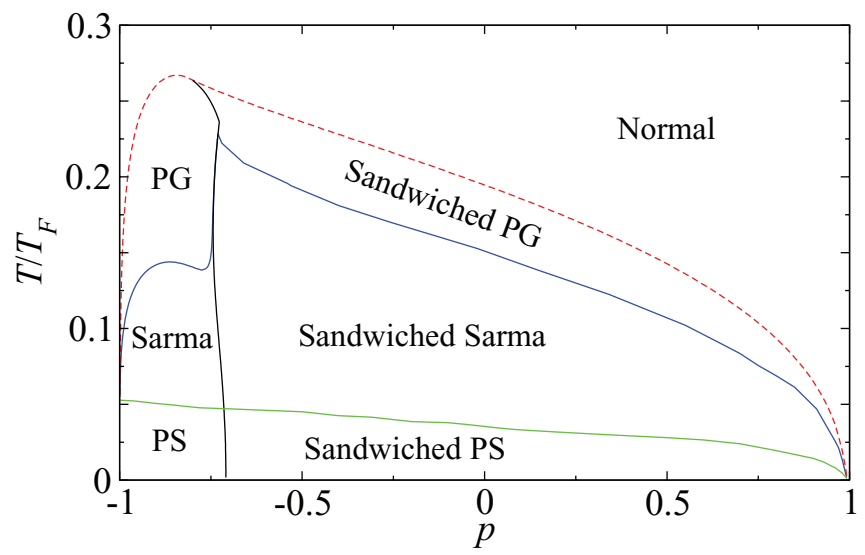

FIG. 1. (Color online) $T-p$ phase diagram of ${ }^{6} \mathrm{Li}^{-40} \mathrm{~K}$ mixture in a harmonic trap at unitarity, with $\omega_{\uparrow}=\omega_{\downarrow}$. The solid lines separate different phases, and the (red) dashed line is approximated by meanfield calculations. Here "PG" and "PS" indicate pseudogapped normal state and phase separation, respectively.

gas counterpart, $\Omega_{N}=-T \sum_{\mathbf{k}, \sigma} \ln \left(1+e^{-\xi_{\mathbf{k} \sigma} / T}\right)$. Here $\Omega_{S}$ consists of contributions from fermionic excitations $\Omega_{F}$ and noncondensed pairs $\Omega_{B}$ :

$$
\begin{aligned}
& \Omega_{S}=\Omega_{F}+\Omega_{B}, \\
& \Omega_{F}=-\frac{\Delta^{2}}{g}+\sum_{\mathbf{k}}\left(\xi_{\mathbf{k}}-E_{\mathbf{k}}\right)-T \sum_{\mathbf{k}, \sigma} \ln \left(1+e^{-E_{\mathbf{k} \sigma} / T}\right), \\
& \Omega_{B}=Z \mu_{p} \Delta_{\mathrm{pg}}^{2}+T \sum_{\mathbf{q}} \ln \left(1-e^{-\tilde{\Omega}_{\mathbf{q}} / T}\right) .
\end{aligned}
$$

The stable states should have a lower value. When $\Omega_{S}>\Omega_{N}$ at certain radii $r$, phase separation takes place. Note that the $\Omega_{B}$ term is absent in simple mean-field calculations.

Shown in Fig. 1 is the calculated $T-p$ phase diagram (for the ${ }^{6} \mathrm{Li}^{40}{ }^{40} \mathrm{~K}$ mixture) at unitarity with $\omega_{\uparrow}=\omega_{\downarrow}$ and $m_{\uparrow} / m_{\downarrow}=$ 40:6. Representative density and gap profiles are shown in Fig. 2. Apparently, phase separation (PS) and sandwiched PS occupy the lowest $T$ part. In particular, when the light species dominates the population, we have a regular phase separation with an equal population superfluid core in the trap center, surrounded by the light atoms, similar to that seen in the equalmass case [29]. Here $\Delta, \Delta_{\mathrm{sc}}$, and $n_{\downarrow}$ jump to zero across the interface, as shown in Fig. 2(c). However, except for the light atom dominated case, a three-shell structure appears, which we refer to as sandwiched PS. As shown in Fig. 2(f), an equal population superfluid exists only at intermediate radii, whereas the light and heavy atoms dominate the outer and inner shells, respectively. In particular, at zero $T$, the heavy atoms are absent in the outer shell. The gaps and density exhibit first-order jumps at both interfaces, consistent with the earlier works at $T=0$ [19]. The primary cause of this three-shell structure is that $R_{\mathrm{TF}}^{\uparrow} \ll R_{\mathrm{TF}}^{\downarrow}$ leads to $n_{\uparrow} \gg n_{\downarrow}$ at the trap center. Furthermore, at low $T$, population imbalance tends to break pairing [30]. Therefore, only at intermediate radii where $n_{\uparrow} \approx$ $n_{\downarrow}$ can a superfluid exist.

In Fig. 1, the PS phase becomes a Sarma phase at intermediate $T$, where population imbalance penetrates into the inner superfluid core so that the first-order jumps at the interface 

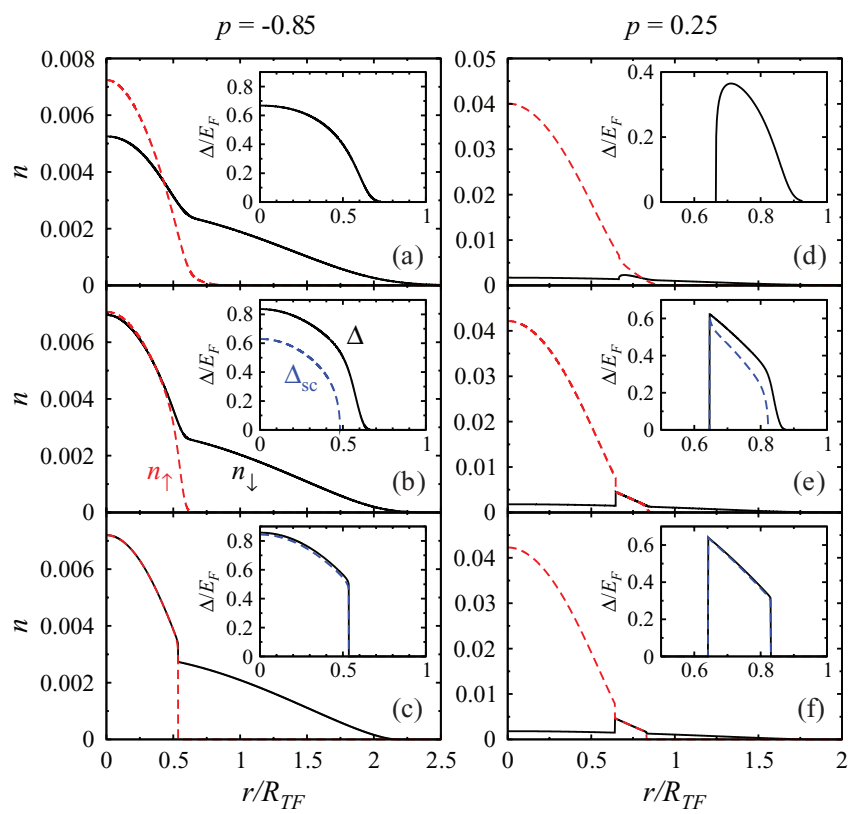

FIG. 2. (Color online) Representative density (main panels) and gap (insets) profiles for $p=-0.85$ and 0.25 at unitarity: (a)-(c) $T / T_{F}=0.2,0.1,0.02$; (d)-(f) $T / T_{F}=0.15,0.05,0.01$, corresponding to each phase in Fig. 1. In (c) and (f), essentially $T=0$. The (black) solid and (red) dashed curves in the main panels are for ${ }^{6} \mathrm{Li}$ ( $n_{\downarrow}$, light species) and ${ }^{40} \mathrm{~K}\left(n_{\uparrow}\right.$, heavy species), respectively. Plotted in the insets are the total gap $\Delta$ (black solid) and order parameter $\Delta_{\mathrm{sc}}$ (blue dashed lines). Here $n_{\sigma}$ is in units of $n_{F} \equiv k_{F}^{3} / 3 \pi^{2}$.

disappear, similar to the Sarma state found in Ref. [29]. Correspondingly, the sandwiched PS phase becomes a sandwiched Sarma phase. As seen from Figs. 2(b) and 2(e), $\Delta, \Delta_{\mathrm{sc}}$, and $n_{\uparrow}$ vanish continuously at the outer interface. The difference between $\Delta$ and $\Delta_{\text {sc }}$ defines the presence of the pseudogap.

At higher $T$, the superfluidity disappears so that the Sarma phase becomes a polarized pseudogap (PG) phase with $\Delta_{\mathrm{pg}} \neq$ 0 in the inner core. Similarly, the sandwiched Sarma phase evolves into a sandwiched PG phase. Representative density and gap profiles are shown in Figs. 2(a) and 2(d). Finally, at very high $T$ we have a normal phase. Note that in Fig. 1 the dashed line separating the normal and the (sandwiched) PG phases indicates a crossover rather than a phase transition.

Figure 3 presents the phase diagrams at (a) $1 / k_{F} a=-0.5$ and (b) 0.5 , similar to Fig. 1, but in the (near-)BCS and (near-)BEC regimes, respectively. For the BCS case, except for the high- $T$ normal phase, the phase diagram is essentially occupied by three-shell structures. The middle shell is an unpolarized BCS superfluid at the lowest $T$, Sarma superfluid at intermediate $T$, and a polarized PG state at slightly higher $T$. As shown in Figs. 4(a) and 4(b), in the sandwiched PS phase, the outer shell is a normal mixture, surrounded by light atoms alone at the trap edge. This is different from the sandwiched PS at unitarity [Fig. 2(f)], where the outer shell contains no normal mixture at $T=0$. In comparison with the unitary case, one can see that the decreased pairing strength squeezes out the PS phase completely. The temperature evolution of the various phases is similar to their unitary counterparts, except that the sandwiched PG phase now occupies a very slim region, reflecting a much weaker pseudogap effect in the BCS regime.

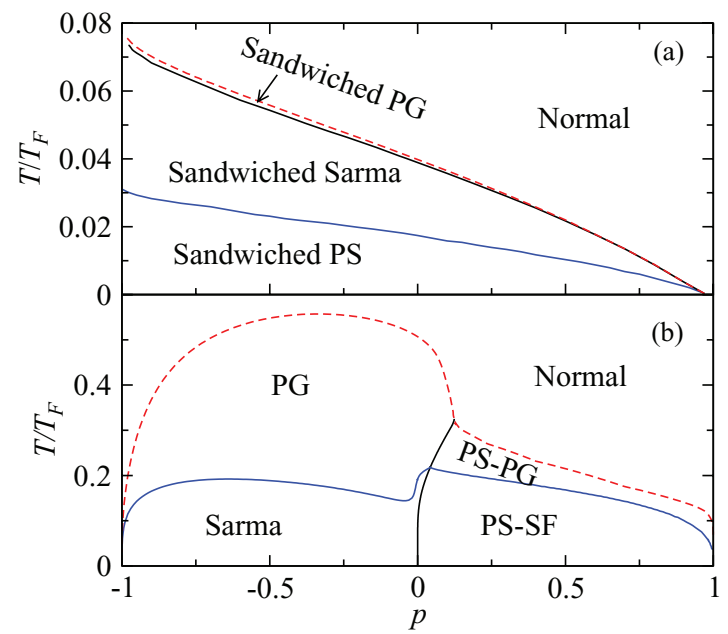

FIG. 3. (Color online) Phase diagram of ${ }^{6} \mathrm{Li}^{-}{ }^{40} \mathrm{~K}$ as in Fig. 1 but for (a) $1 / k_{F} a=-0.5$ and (b) +0.5 . Here "PS-SF" and "PS-PG" represent phase-separated superfluid and phase-separated pseudogap phases, respectively, with a normal gas core surrounded by an outer shell of superfluid or pseudogapped normal mixture.

The phase diagram for the BEC case in Fig. 3(b) is rather different. First, for $p<0$, where the light species dominates, a Sarma superfluid phase occurs at low $T$. Indeed, polarized superfluid becomes stable in the BEC regime $[6,30]$. As $T$ increases, $\Delta_{\text {sc }}$ vanishes and the system evolves into a polarized pseudogap state. The large area of the "PG" phase indicates greatly enhanced pseudogap effects in the BEC regime. On the other hand, for (roughly) $p>0$, we have an "inverted" phase separated superfluid state at low $T$, labeled as "PS-SF," where a normal gas core of the heavy species is surrounded by a shell of unpolarized superfluid. This should be contrasted with the PS phase in the unitary case [Fig. 2(c)], where the normal Fermi gas is outside the superfluid core.
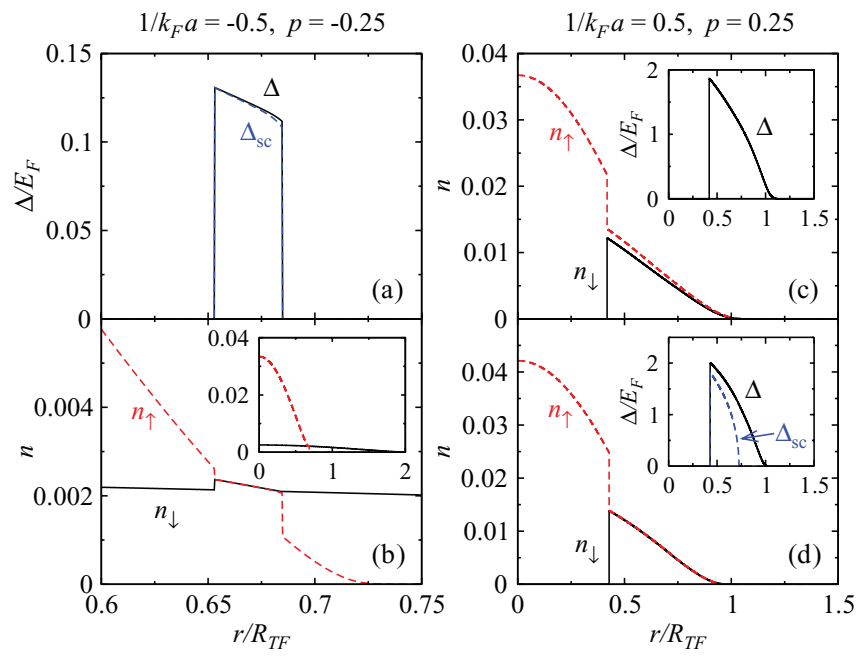

FIG. 4. (Color online) Typical density and gap profiles for BCS (left column, $1 / k_{F} a=-0.5$ ) and BEC (right column, $1 / k_{F} a=0.5$ ) regimes. The convention is the same as in Fig. 2. Panels (a) and (b) are for $p=-0.25$ at $T=0.01 T_{F} \approx 0$, corresponding to the sandwiched PS phase in Fig. 3(a). Panels (c) and (d) plot the density and gap (insets) distributions for $p=0.25$ at $T=0.2 T_{F}$ and $0.1 T_{F}$, representing the PS-PG and PS-SF phases in Fig. 3(b), respectively. 


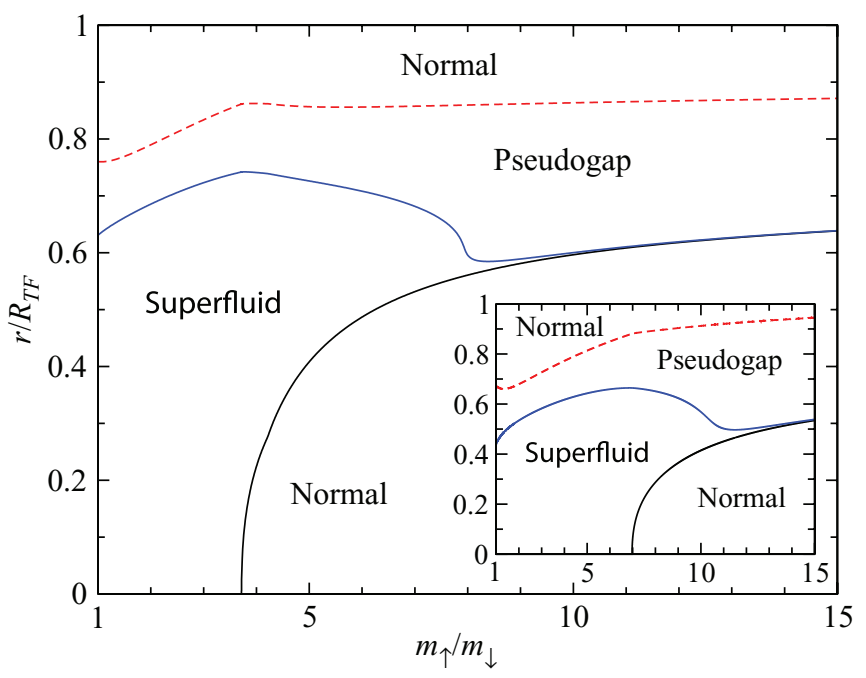

FIG. 5. (Color online) Temperature sensitive spatial distributions of various phases in the trap at unitarity as a function of mass ratio $m_{\uparrow} / m_{\downarrow}$ for $p=-0.23$ with $T=0.1 T_{F}, \omega_{\uparrow}=\omega_{\downarrow}$ (main figure) and $T=0.15 T_{F}, \omega_{\downarrow}=2 \omega_{\uparrow}$ (inset).

As $T$ increases, a phase-separated pseudogap state (labeled "PS-PG") appears, where pseudogap exists in the polarized outer shell but without superfluidity. This is an exotic new phase in that in a homogeneous system the evolution with increasing $T$ from a pseudogap state to the unpaired normal state is a crossover rather than a phase transition. It is only for energetic reasons that at the same intermediate $T$ in the presence of trap inhomogeneity does such phase separation occur in real space. Across the phase boundaries between the Sarma and PS-SF and between PG and PS-PG phases, the densities undergo dramatic restructuring. Typical density and gap profiles for the PS-PG and PS-SF phases are shown in Figs. 4(c) and 4(d), respectively. Such phase separation (as well as the sandwiched PG state) have never been predicted or experimentally observed before. Possible causes for the "inversion" of the phase separation include (i) for $p>0$, $R_{\mathrm{TF}}^{\uparrow}$ of the heavy species becomes close to $R_{\mathrm{TF}}^{\downarrow}$ of the light atoms; and (2) as $k_{F}(r)$ decreases with $r$, the outer region is deeper in the BEC regime than the trap center, making pairing energetically more favorable at the trap edge. As the pairing strength increases from unitarity, the outer shell of normal light atoms of the three-shell structure retreats and finally disappears.
We now turn to the case of variable $m_{\uparrow} / m_{\downarrow}$, with different $\omega_{\uparrow} / \omega_{\downarrow}$. Plotted in Fig. 5 are spatial distributions of possible phases at unitarity as a function of the mass ratio at $p=-0.23$ for $\left(T / T_{F}, \omega_{\uparrow} / \omega_{\downarrow}\right)=(0.1,1)$ (main figure) and $(0.15,1 / 2)$ (inset), respectively, due to the presence of the trap. For both cases, a sandwichlike structure appears as $m_{\uparrow} / m_{\downarrow}$ increases beyond 3.7 and 7.0, respectively. Indeed, for $m_{\uparrow} / m_{\downarrow}=1$ and $\omega_{\uparrow} / \omega_{\downarrow}=1$, pairing is always easier at $r=0$ than elsewhere. However, as $m_{\uparrow} / m_{\downarrow}$ becomes sufficiently large, $n_{\sigma}(r)$ crosses each other at an intermediate radius, so that a three-shell structure appears at low $T$. Apparently a large mass imbalance makes population balance or imbalance less important. Note that BCS pairing requires a match of (mass independent) $k_{F}^{\sigma}=\left(6 \pi^{2} n_{\sigma}\right)^{1 / 3}$ (locally) between the two species. The radius of density crossing may be tuned, via changing $R_{\mathrm{TF}}^{\sigma}$, by either $m_{\uparrow} / m_{\downarrow}$ or $\omega_{\uparrow} / \omega_{\downarrow}$ or both, since $R_{\mathrm{TF}}^{\sigma}$ depends on the product $m_{\sigma} \omega_{\sigma}$. As shown in the inset, the threshold $m_{\uparrow} / m_{\downarrow}$ for the three-shell structure to occur is roughly doubled when $\omega_{\uparrow} / \omega_{\downarrow}$ is reduced by $1 / 2$. While the difference between different $\omega_{\uparrow} / \omega_{\downarrow}$ is mainly quantitative, for the ${ }^{6} \mathrm{Li}^{40} \mathrm{~K}$ mixture, a three-shell structure appears in the main figure, while only a regular Sarma phase shows up in the inset.

Finally, the neglected incoherent part of the self-energy $\delta \Sigma_{\sigma}$ may induce polarons in the mixed normal states, which, however, is unimportant for the present study. Following common practice $[2,3,25,31-35]$, we have also dropped the particle-hole channel contributions [36], which can be roughly approximated by a shift in $1 / k_{F} a[37,38]$. These approximations are expected to influence the phase boundaries only quantitatively. In addition, the FFLO states, which appear to be of less interest in a 3D equal-mass Fermi gas [39-42], will be investigated in a future work.

We end by noting that the widespread pseudogap phenomena, which are unique to our theory, and the prediction of exotic phases, e.g., the phase-separated pseudogap phase, can be tested using vortex measurements [43] and rf spectroscopy $[27,44]$, etc. Comparison with concrete experiments using detailed parameters such as $N_{\sigma}, \omega_{\sigma}$ will be possible when such experiments become available in the (near) future.

This work is supported by the National Basic Research Program of China (Grants No. 2011CB921303 and No. 2012CB927404), NSF of China (Grants No. 10974173 and No. 11274267), and Fundamental Research Funds for Central Universities of China (Program No. 2010QNA3026).
[1] T. Timusk and B. Statt, Rep. Prog. Phys. 62, 61 (1999).

[2] A. J. Leggett, Modern Trends in the Theory of Condensed Matter (Springer-Verlag, Berlin, 1980), pp. 13-27.

[3] P. Nozières and S. Schmitt-Rink, J. Low Temp. Phys. 59, 195 (1985).

[4] Q. J. Chen, J. Stajic, S. N. Tan, and K. Levin, Phys. Rep. 412, 1 (2005).

[5] S. Giorgini, L. P. Pitaevskii, and S. Stringari, Rev. Mod. Phys. 80, 1215 (2008).

[6] Q. J. Chen, Y. He, C.-C. Chien, and K. Levin, Phys. Rev. A 74, 063603 (2006).
[7] G. Sarma, J. Phys. Chem. Solids 24, 1029 (1963).

[8] P. Fulde and R. A. Ferrell, Phys. Rev. 135, A550 (1964); A. I. Larkin and Y. N. Ovchinnikov, Zh. Eksp. Teor. Fiz. 47, 1136 (1964) [Sov. Phys. JETP 20, 762 (1965)].

[9] D. J. MacNeill and F. Zhou, Phys. Rev. Lett. 106, 145301 (2011); C. J. M. Mathy, M. M. Parish, and D. A. Huse, ibid. 106, 166404 (2011).

[10] E. Wille, F. M. Spiegelhalder, G. Kerner, D. Naik, A. Trenkwalder, G. Hendl, F. Schreck, R. Grimm, T. G. Tiecke, J. T. M. Walraven et al., Phys. Rev. Lett. 100, 053201 (2008); F. M. Spiegelhalder, A. Trenkwalder, D. Naik, G. Hendl, 
F. Schreck, and R. Grimm, ibid. 103, 223203 (2009); F. M. Spiegelhalder, A. Trenkwalder, D. Naik, G. Kerner, E. Wille, G. Hendl, F. Schreck, and R. Grimm, Phys. Rev. A 81, 043637 (2010); A. Trenkwalder, C. Kohstall, M. Zaccanti, D. Naik, A. I. Sidorov, F. Schreck, and R. Grimm, Phys. Rev. Lett. 106, 115304 (2011); D. Naik, A. Trenkwalder, C. Kohstall, F. Spiegelhalder, M. Zaccanti, G. Hendl, F. Schreck, R. Grimm, T. Hanna, and P. Julienne, Eur. Phys. J. D 65, 55 (2011); C. Kohstall, M. Zaccanti, M. Jag, A. Trenkwalder, P. Massignan, G. M. Bruun, F. Schreck, and R. Grimm, Nature (London) 485, 615 (2012).

[11] M. Taglieber, A.-C. Voigt, T. Aoki, T. W. Hänsch, and K. Dieckmann, Phys. Rev. Lett. 100, 010401 (2008); A.-C. Voigt, M. Taglieber, L. Costa, T. Aoki, W. Wieser, T. W. Hänsch, and K. Dieckmann, ibid. 102, 020405 (2009); 105, 269904 (2010); L. Costa, J. Brachmann, A.-C. Voigt, C. Hahn, M. Taglieber, T. W. Hänsch, and K. Dieckmann, ibid. 105, 123201 (2010); 105, 269903 (2010).

[12] T. G. Tiecke, M. R. Goosen, A. Ludewig, S. D. Gensemer, S. Kraft, S. J. J. M. F. Kokkelmans, and J. T. M. Walraven, Phys. Rev. Lett. 104, 053202 (2010).

[13] M. Iskin and C. A. R. Sá de Melo, Phys. Rev. A 77, 013625 (2008).

[14] D. Blume, Rep. Prog. Phys 75, 046401 (2012).

[15] J. E. Baarsma, J. Armaitis, R. A. Duine, and H. T. C. Stoof, Phys. Rev. A 85, 033631 (2012); P. Massignan, Europhys. Lett. 98, 10012 (2012); J.-L. Song and F. Zhou, Phys. Rev. A 84, 013601 (2011).

[16] K. M. Daily and D. Blume, Phys. Rev. A 85, 013609 (2012).

[17] M. Iskin and C. A. R. Sá de Melo, Phys. Rev. Lett. 97, 100404 (2006).

[18] M. M. Parish, F. M. Marchetti, A. Lamacraft, and B. D. Simons, Phys. Rev. Lett. 98, 160402 (2007).

[19] G.-D. Lin, W. Yi, and L.-M. Duan, Phys. Rev. A 74, 031604 (2006).

[20] S.-T. Wu, C.-H. Pao, and S.-K. Yip, Phys. Rev. B 74, 224504 (2006); C.-H. Pao, S.-T. Wu, and S.-K. Yip, Phys. Rev. A 76, 053621 (2007).

[21] T. Paananen, P. Törmä, and J.-P. Martikainen, Phys. Rev. A 75, 023622 (2007).
[22] H. Guo, C.-C. Chien, Q. J. Chen, Y. He, and K. Levin, Phys. Rev. A 80, 011601 (2009).

[23] K. B. Gubbels, J. E. Baarsma, and H. T. C. Stoof, Phys. Rev. Lett. 103, 195301 (2009); J. E. Baarsma, K. B. Gubbels, and H. T. C. Stoof, Phys. Rev. A 82, 013624 (2010).

[24] Q. J. Chen, Y. He, C.-C. Chien, and K. Levin, Phys. Rev. B 75, 014521 (2007).

[25] Q. J. Chen, I. Kosztin, B. Jankó, and K. Levin, Phys. Rev. Lett. 81, 4708 (1998).

[26] J. Stajic, J. N. Milstein, Q. J. Chen, M. L. Chiofalo, M. J. Holland, and K. Levin, Phys. Rev. A 69, 063610 (2004).

[27] J. T. Stewart, J. P. Gaebler, and D. S. Jin, Nature (London) 454, 744 (2008).

[28] Q. J. Chen and K. Levin, Phys. Rev. Lett. 102, 190402 (2009).

[29] C. C. Chien, Q. J. Chen, Y. He, and K. Levin, Phys. Rev. Lett. 98, 110404 (2007).

[30] C. C. Chien, Q. J. Chen, Y. He, and K. Levin, Phys. Rev. Lett. 97, 090402 (2006).

[31] J. R. Engelbrecht, M. Randeria, and C. A. R. Sáde Melo, Phys. Rev. B 55, 15153 (1997).

[32] A. Perali, P. Pieri, L. Pisani, and G. C. Strinati, Phys. Rev. Lett. 92, 220404 (2004).

[33] Y. Ohashi and A. Griffin, Phys. Rev. A 72, 013601 (2005).

[34] H. Hu, X. J. Liu, and P. D. Drummond, Europhys. Lett. 74, 574 (2006).

[35] R. Haussmann, Z. Phys. B 91, 291 (1993).

[36] L. P. Gorkov and T. K. Melik-Barkhudarov, Sov. Phys. JETP 13, 1018 (1961).

[37] Z.-Q. Yu, K. Huang, and L. Yin, Phys. Rev. A 79, 053636 (2009).

[38] Q. J. Chen, arXiv:1109.2307.

[39] Y. He, C.-C. Chien, Q. J. Chen, and K. Levin, Phys. Rev. A 75, 021602 (2007).

[40] D. E. Sheehy and L. Radzihovsky, Phys. Rev. Lett. 96, 060401 (2006).

[41] W. Zhang and L.-M. Duan, Phys. Rev. A 76, 042710 (2007).

[42] We note that the supersolid phase discussed in Ref. [23] is just the LO state.

[43] M. W. Zwierlein, J. R. Abo-Shaeer, A. Schirotzek, and W. Ketterle, Nature (London) 435, 170404 (2005).

[44] Q. J. Chen, Y. He, C.-C. Chien, and K. Levin, Rep. Prog. Phys. 72, 122501 (2009). 